\title{
Resonant efficiency improvement design of piezoelectric biosensor for bacteria gravimetric sensing
}

\author{
Jang-Zern Tsai ${ }^{\mathrm{a}}$, Ching-Jung Chen ${ }^{\mathrm{b}, *}$, Dung-Ting Shie ${ }^{\mathrm{a}}$ and Jen-Tsai Liu ${ }^{\mathrm{c}, *}$ \\ ${ }^{a}$ Department of Electrical Engineering, National Central University, No.300, Jhongda Rd., Jhongli \\ City, Taoyuan County 32001, Taiwan, R.O.C. \\ ${ }^{b}$ School of Electronic, Electrical and Communication Engineering, University of Chinese Academy of \\ Sciences, No.19A Yuquan Road, Beijing 100049, China \\ ${ }^{c}$ The College of Materials Sciences and Opto-Electronic Technology, University of Chinese Academy \\ of Sciences, No.19A Yuquan Road, Beijing 100049, China
}

\begin{abstract}
The piezoelectric biosensor have been widely used in ultra-small mass detection of biomolecular, based on PZT piezoelectric material can create a variety of compositions geometrically; it could widely develop a high-frequency resonator and measure the change of the slightest mass while improve the limited detection simultaneously. Therefore, the piezoelectric biosensor of this study was fabricated by a spin-coating method and backside etching process for improving the characteristic of piezoelectric biosensor. The result exhibited that the $250 \mu \mathrm{m} \times 250 \mu \mathrm{m}$ working size has the most favorable piezoelectric characteristic. The tunability was approximately $38.56 \%$ and it showed that reducing the substrate thickness could obtain a clear resonance signal in a range of 60 to $380 \mathrm{MHz}$. In theory calculated for gravimetric sensing, it could achieve $0.1 \mathrm{ng}$ sensing sensitivity. In gravimetric sensing, the sensing range was between $50,000 \sim 100,000 \mathrm{CFU} / \mathrm{ml}$. Sensing range was lower in clinical urinary tract infection $(100,000 \mathrm{CFU} / \mathrm{ml})$, thus demonstrating its usefulness for preventive medicine. It can understand the piezoelectric sensor of this study has potential application in the future for biomedical gravimetric sensing.
\end{abstract}

Keywords: Lead zirconate titanate (PZT), piezoelectric, biosensor, resonance-frequency, escherichia coli (E. coli)

\section{Introduction}

In recent years, many biosensors have been widely used in ultra-small mass detection [1-3], such as surface plasmon resonance (SPR), guided mode resonance (GMR) and piezoelectric biosensor. SPR and GMR are based on refraction change to calculate the loading mass. However, piezoelectric biosensor is based on mass loading to change resonant frequency; it can understand piezoelectric

\footnotetext{
${ }^{*}$ Corresponding authors: Ching-Jung Chen, School of Electronic, Electrical and Communication Engineering, University of Chinese Academy of Sciences, No.19A Yuquan Road, Beijing 100049, China. Tel.: +86-13261037737; Fax: +86-01088256701; E-mail: cjchen@ucas.ac.cn.

Jen-Tsai Liu, The College of Materials Sciences and Opto-Electronic Technology, University of Chinese Academy of Sciences, No.19A Yuquan Road, Beijing 100049, China. Tel.: +86-15652782159; Fax: +86-010-88256701; E-mail: jtliu@ucas.ac.cn.
}

0959-2989/14/\$27.50 @ 2014 - IOS Press and the authors. 
biosensor suitable for gravimetric sensing more than other sensors. However the piezoelectric biosensor development is limited by how to increase the fundamental frequency. The resonant frequency refers to the type of dependence that Sauerbrey claimed existed in a relationship between variables required to determine the mass changes in thin films. The findings are summarized in the following equation [4]:

$$
\Delta f=\frac{-2 f_{0}^{2}}{A \sqrt{\rho \times \mu}} \Delta m=K f_{0}^{2} \Delta m
$$

$f_{0}$ is the resonant of the fundamental frequency; the density and shear modulus of piezoelectric materials are and $\mu$, respectively. The resonance area is $A$. Frequency change is $\Delta f$. Mass of change on the sensor surface is $\Delta_{m}$ and $K$ is constant. Based on Sauerbrey's equation, the square of fundamental frequency proportioned to $1 / \Delta_{m}$. But the sensitivity is related to frequency change. Thus, the sensitivity can be rewritten:

$$
\text { Sensitivity }=\Delta f /(\Delta m / A)
$$

It is well know the limitations of micro-balances biosensor is low resonant frequency. Therefore, to develop a high frequency micro-balance biosensor for overcoming the limitation and improving the detection limitation are desperately need. Piezoelectric materials generate a voltage in response to an applied force, and this property is reversible. Similarly, the piezoelectric materials deform with an applied voltage. As they can generate a slight force and vibration that have different practical applications as actuators, sensors, motors, pumps, and transducers [4-8]. The piezoelectric device has also been widely used in motorized, biomedical, industrial measurements, automatic control, and other electronic products. Lead zirconium titanate (PZT) is a popular piezoelectric material, because it can design a variety of compositions geometrically and has a high capacitance property, high electromechanical coupling coefficients, temperature stability, high resistance to depolarization from mechanical stress, and high driving voltages. Recently, many deposition methods of PZT thin film have been developed. Among the different methods of deposition, the sol-gel method has the advantage of low cost, more favorable stoichiometry, and simplicity. Utilizing the sol-gel deposition process, the PZT have morphotropic phase boundary composition that exists the rhombohedral phase and tetragonal phase. Therefore, it exhibits numerous and varied polarization orientations resulting in excellent electromechanical properties.

In this study, it examined PZT layers with micrometer working sizes by a spin-coating method and backside etching process for improving the resonant efficiency of piezoelectric biosensor. The backside etchings were constructed from piezoelectric freestanding thin films that exhibited electrostrictive effect enhanced sensitivity of surface mass sensing. In order to enhance the PZT crystalline structure, the annealing conditions were also investigated. Through these experiments, this study will get the optimal conditions for PZT sensor development. Finally, the biomass detection also tests by loading Escherichia coli $\mathrm{O} 157: \mathrm{H} 7$ at different concentrations between $10000 \mathrm{cells} / \mathrm{ml}$ to $100000 \mathrm{cells} / \mathrm{ml}$. Through above study will develop this sensor have potential application in future on biomedical detections, especially in the clinical urinary tract infection for preventive medicine. 


\section{Research methodology}

\subsection{Fabrication of PZT piezoelectric sensor}

Before the PZT piezoelectric sensor fabrication, the chip used the chemical thermal etching method to pull out the backside of the PZT working area. First, the silicon dioxide $(500 \mathrm{~nm})$ was grown on both sides of the silicon using thermal oxidation, followed by deposition of $100 \mathrm{~nm}$ silicon nitride by LPCVD. On the backside, the high density plasma (HDP)-etched nitride layer with a positive photoresist as a mask and oxide layer was patterned using a buffered oxide etcher (BOE) with the same photoresist mask layer. Using a $30 \%$ potassium hydroxide solution etched silicon substrate at $80^{\circ} \mathrm{C}$ along with mild stirring for $10 \mathrm{~h}$. After the silicon etching, the nitride layer was removed using hot phosphoric acid. Finally, the backside of the chip was etched approximately $0.3 \mu \mathrm{m}$ diameter.

After backside etching, the chip includes two PZT piezoelectric devices and fabricates the sandwich structure by micro-photolithography technology. This process used the single side polished, p-type (100) 4" silicon wafers with $525 \pm 25 \mu$ m thickness for device fabrication. In addition, the $500 \mathrm{~nm} \mathrm{SiO}_{2}$ thick layer was manufactured by thermal oxidation between the silicon substrate and the bottom electrode layer, serving as a diffusion barrier. On the upper side, the Pt bottom electrode and Ti adhesive were patterned by a lift-off technique and deposited by an E-gun evaporator. The PZT piezoelectric thin film was then coated by a spinner, and this high concentration sol-gel PZT material $(110 / 52 / 48)$ was produced by Kojundo Chemical Lab. Corp., Japan. After each coating, the PZT thin film was dried at $180^{\circ} \mathrm{C}$ and pyrolysis at $350^{\circ} \mathrm{C}$ for five min. After that the PZT thin film was formulated by lithography.

\subsection{Characterization of PZT piezoelectric sensor}

The PZT thin film properties were verified by optical microscopy (OM) and field emission scanning electron microscopy (FESEM, Hitatchi S-4300). The PZT thin film was crystallized using a furnace system, and the crystallization was transferred to the single-phase peroviskite structure and identified by X-ray diffraction (XRD, D8 advanced). The XRD patterns were recorded at a scan size of $0.05^{\circ}$ for phase analysis. Using the aforementioned method, the optimal PZT piezoelectric sensor fabrication process could be determined.

Due to comparing different working area sizes of PZT piezoelectric sensors using the same fabrication process, the PZT piezoelectric device was designed four types of area size $(50 \mu \mathrm{m} \times 50 \mu \mathrm{m}, 100$ $\mu \mathrm{m} \times 100 \mu \mathrm{m}, 250 \mu \mathrm{m} \times 250 \mu \mathrm{m}$, and $400 \mu \mathrm{m} \times 400 \mu \mathrm{m})$. This paper discusses which area size of piezoelectric sensor has the most favorable piezoelectric characteristic. However, the capacitance properties of all PZT sensors were observed using HP 4284 LCR meter and Keithley 4200 semiconductor analyzer/dc voltage source. Finally, the PZT sensor was used the HP 4191A impedance analyzer to measure the resonance ( $\mathrm{fr}$ ) and anti-resonance frequency (fa) of the PZT piezoelectric sensor, which could identify the resource characteristic of different backside fabrications of the PZT piezoelectric sensor.

\subsection{Bacteria gravimetric sensing}

Luria-Bertani broth (LB broth) is a medium for bacteria culture because it demonstrates fast growth and favorable growth yields. Therefore, the LB broth medium of the culture experiment was prepared using purified water with frequent agitation and an autoclave at $121^{\circ} \mathrm{C}$ for $25 \mathrm{~min}$ in the first step. In 


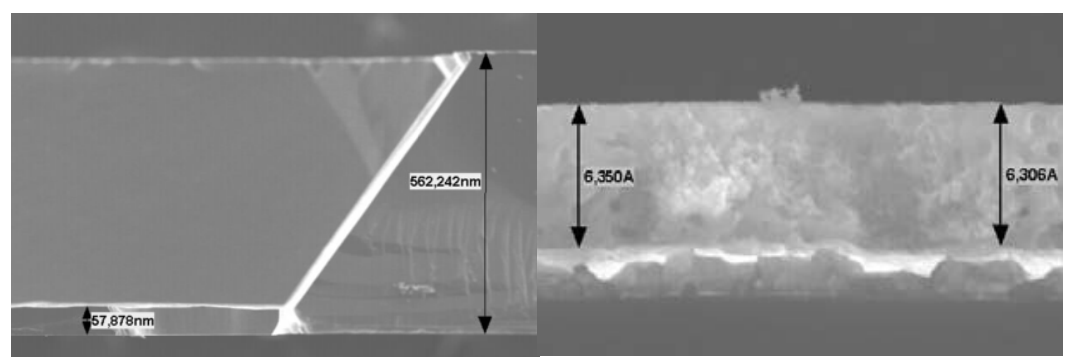

Fig. 1. SEM micrographs: (a) Cross-sectional profiles of the diaphragm (b) cross-sectional of the PZT thin film.

the second step, the culture of E. coli was grown at $37^{\circ} \mathrm{C}$ for 12 hours with mild stirring in LB broth, then it was measured the optical density at $600 \mathrm{~nm}\left(\mathrm{OD}_{600}\right)$, wavelength approximately $10^{9} \mathrm{CFU} / \mathrm{ml}$. The concentration conditions of $E$. coli $\left(10^{8}, 10^{7}, 10^{6}, 10^{5}\right.$, and $10^{4}$ cells $\left./ \mathrm{mL}\right)$ were also prepared in a culture medium using accurate serial dilution.

After different cell number conditions of E. coli preparation [9], one microliter of a different cell number condition was loaded on the PZT chip, covering the sensing area. Simultaneously, the impedance and phase angle of the PZT resonant peak were monitored directly. Finally, this study recorded a constant change in resonant frequency; this signal change was due to mass change of different cell numbers.

\section{Results and discussion}

\subsection{Characterization of PZT piezoelectric sensor}

The piezoelectric sensor was manufactured using a micro-photolithography method. The whole chip was manufactured using a silicon wafer and diaphragm $0.3 \mathrm{~mm}$ in length and $50 \mu \mathrm{m}$ in thickness. The PZT layer was manufactured between the bottom electrode layer and the top electrode. The bottom and top electrodes consisted of platinum $(\mathrm{Pt})$ and tantalum $(\mathrm{Ta})$ films $(100$ and $50 \mathrm{~nm}$ thick, respectively), and gold ( $\mathrm{Au}$ ) and tantalum (Ta) films (100 and $50 \mathrm{~nm}$ thick, respectively). In addition, the backside hole was etched opposite the PZT piezoelectric sensor area, directly affecting the PZT piezoelectric sensor resource efficiency. The cross-sectional profiles of the diaphragms are shown in Figure 1(a). The cross-section of the PZT piezoelectric films is shown in Figure 1(b) with the SEM micrographs. The film shows grain and void-free structures; the thickness from five cycles of drying and sintering is approximately $630 \mathrm{~nm}$.

\subsection{Crystallization and micrograph of the PZT thin film}

In order to determine the optimal temperature of crystalline phase transfer, the crystalline phases of the PZT thin film were manufactured with various annealing conditions. The primary function of the annealing process involves facilitating the amorphous phase crystallization. First, this study changed the annealing temperature from $500^{\circ} \mathrm{C}$ to $900^{\circ} \mathrm{C}$ and kept all annealing times at $20 \mathrm{~min}$. All results identified by X-ray diffraction patterns of different temperatures and SEM cross-sectional profiles of the thin films are shown in Figure 2(a). Based on previous reports [10], the combination of (1 000$),(1$ $10),\left(\begin{array}{lll}1 & 1 & 1\end{array}\right),\left(\begin{array}{lll}2 & 0 & 0\end{array}\right)$, and $\left(\begin{array}{lll}2 & 1 & 1\end{array}\right)$ crystal planes is indicated by the (111)-preferred orientation perovskite phase. Regarding the near morphotropic phase boundary (MPB), the (111) texture of 


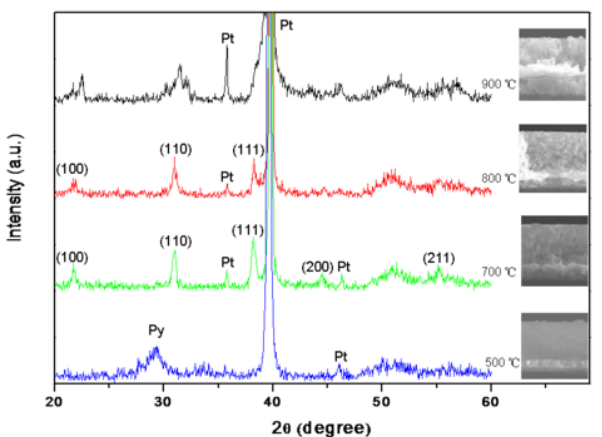

(a)

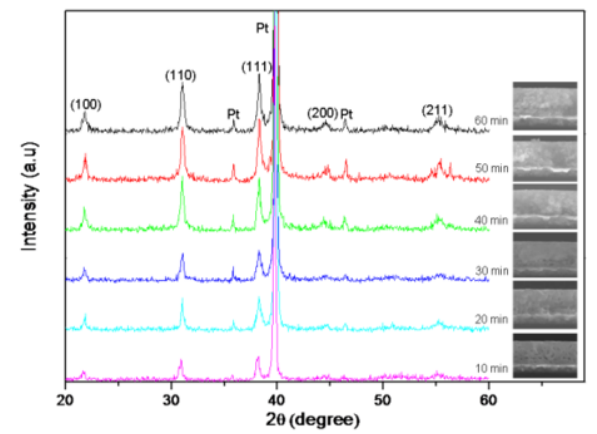

(b)

Fig. 2. XRD patterns of PZT thin films with various annealing conditions: (a) annealing at different temperatures; (b) annealing at $700^{\circ} \mathrm{C}$ with different times.

crystalline phases of the PZT thin film apparently improved considerably the ferroelectric property and the (111) texture was provided the largest polarization with the rhombohedral phase $[11,12]$. The XRD profile and the SEM show the PZT thin film has pyrochlore phase and no crystalline PZT of the PZT film sintering at $500^{\circ} \mathrm{C}$. Therefore, the PZT crystalline at $500^{\circ} \mathrm{C}$ can ${ }^{\prime} t$ transfer PZT thin film to the perovskite phase due to the annealing temperature being too low [13]. However, accompanies the annealing temperature increased $\left(800^{\circ} \mathrm{C}\right.$ and $\left.900^{\circ} \mathrm{C}\right)$, the $(100)(111)$ peaks was weak than $700^{\circ} \mathrm{C}$ and the peak of (200) also disappeared. This phenomenon was due to the excessive loss of lead from the PZT and provoking the perovskite phase to convert to the $\mathrm{PbTi}_{3} \mathrm{O}_{7}$ phase [14]. Therefore, the optimal annealing temperature of $700^{\circ} \mathrm{C}$ could be confirmed. To enhance the particular perovskite phase and obtain the higher piezoelectric constant, the PZT thin film was manufactured at $700^{\circ} \mathrm{C}$ annealing temperature for different durations. All tests were then identified using X-ray diffraction pattern, such as shown in Figure $2(\mathrm{~b})$. Under the $700^{\circ} \mathrm{C}$ annealing condition with different annealing times, the PZT thin films all obtained an individual ferroelectric perovskite phase. Between 10 and $40 \mathrm{~min}$, the intensity of the single perovskite peaks increased as the annealing time and the void of PZT thin film improved. The contrasts with the SEM photograph, due to increased crystallization through postannealing [15]. Moreover, when the annealing time was higher than $40 \mathrm{~min}$ (40 $\mathrm{min}$ to $60 \mathrm{~min}$ ), the peak intensity of the perovskite phase did not change, as shown in Figure 2(b). Finally, PZT thin film solidified using the annealing process at $700^{\circ} \mathrm{C}$ for $40 \mathrm{~min}$ in this study.

\subsection{Capacitance-voltage and resonant property analysis}

The piezoelectric property of the PZT sensors can be observed by C-V curve measuring [16]. Due to the PZT lattice strain, the electrical field can cause the PZT thin film to be polarized and can define the capacitance by the $\mathrm{C}=\varepsilon \mathrm{A} / \mathrm{d}$ equation (where $\varepsilon$ is the dielectric constant, $\mathrm{d}$ is the distance of the electrodes and $\mathrm{A}$ is the piezoelectric area). Therefore, the capacitance value is an important result for exhibiting the relative permeability of lattice strain in PZT sensor. The $\mathrm{C}-\mathrm{V}$ measurement with different PZT piezoelectric working PZT sensors areas was applied -8 to $8 \mathrm{~V}$ DC bias using an HP 4284 LCR meter and a Keithley 4200 semiconductor analyzer/dc voltage source; the result is shown in Figure 3(a). In Figure 3(a), the various electrical fields induced the different capacitance values having two maxima sharp that corresponded to the coercive fields. This curve indicates the lattice of piezoelectric film having polarization displacement, as well as the butterfly loops [17]. In this curve, the capacitance maximal value and the lowest value of the butterfly curve also represent the 


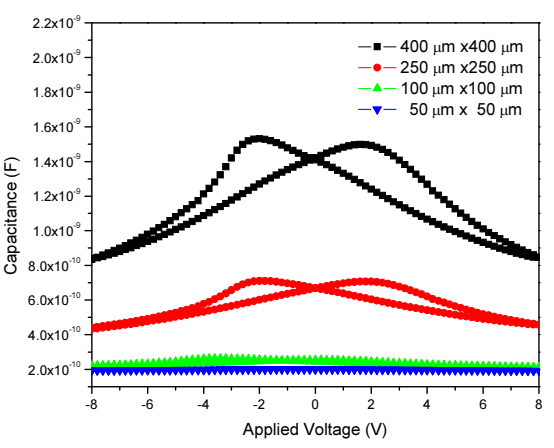

(a)

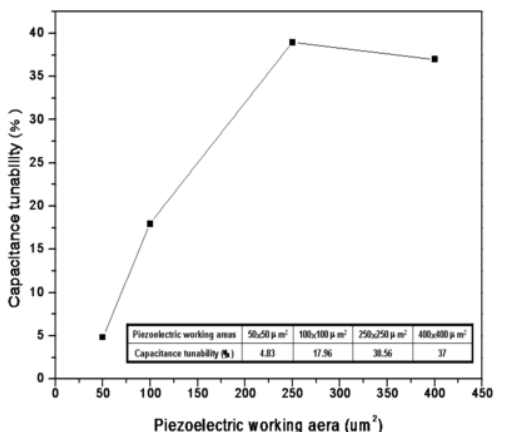

(b)

Fig. 3. (a) The C-V curves of the various PZT piezoelectric working areas; (b) The relationship between capacitance tunability and the different PZT piezoelectric working areas.

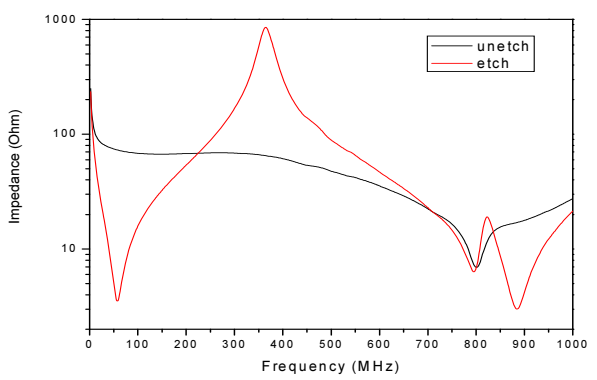

(a)

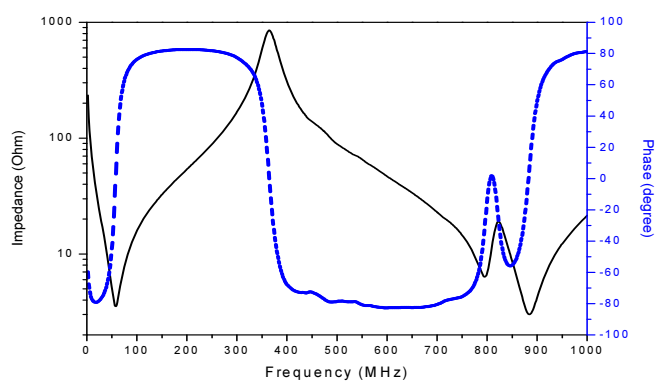

(b)

Fig. 4. (a) Impedance properties of un-etch and after etched $250 \mu \mathrm{m} \times 250 \mu \mathrm{m}$ PZT sensor. (b) Impedance and phase curve of after etched $250 \mu \mathrm{m} \times 250 \mu \mathrm{m}$ PZT sensor.

property of polarization switching and denotes tenability $[18,19]$. Therefore, the capacitance tunability percentage can be defined by $\left[\left(C_{\max }-C_{\min }\right) / C_{\max }\right] \times 100$. As shown in Figure $3(b)$, the $50 \mu \mathrm{m} \times 50 \mu \mathrm{m}$ PZT device has the lowest tunability of approximately $4.83 \%$, and the $250 \mu \mathrm{m} \times 250 \mu \mathrm{m}$ PZT device has the highest tunability of approximately $38.56 \%$. It is a linear relationship of the capacitance tunability and piezoelectric working area from $50 \mu \mathrm{m}^{2}$ to $250 \mu \mathrm{m}^{2}\left(\mathrm{R}^{2}=0.98\right)$ and the tenability less decreases at $400 \mu \mathrm{m} \times 400 \mu \mathrm{m}$ PZT device is approximately $37 \%$. Therefore, the result could be compared with the leakage density current and C-V properties. The $250 \mu \mathrm{m} \times 250 \mu \mathrm{m}$ PZT sensor has favorable insulation and excellent piezoelectric characteristic performance. Therefore, the $250 \times 250$ $\mu \mathrm{m}^{2}$ PZT sensor is the optimal sensor for applications in all subsequent experiments.

The resonance frequency of the PZT device was measured by an impedance analyzer (HP 4191A). The range of frequency sweeps from $1 \mathrm{MHz}$ to $1000 \mathrm{MHz}$ and the step size is $1 \mathrm{MHz}$. Figure 4(a) shows the impedance characteristic of $250 \times 250 \mu \mathrm{m}^{2}$ PZT sensors via un-etched and etched treatment with different frequencies. Generally, the resonance signal could be determined by the impedance characteristic measurement, and has two frequency points of zero phases. The series resonance occurred at the first frequency. At this frequency point, the PZT piezoelectric chip appeared resistive in the circuit; impedance and current flow was at the minimum and maximum, respectively. As the frequency increased beyond the series resonance point, the PZT piezoelectric chip appeared to be inductive in the circuit. When the reactance of the motional inductance and shunt capacitance canceled, the PZT piezoelectric chip was at this point, and the impedance and current flow was maximized and 


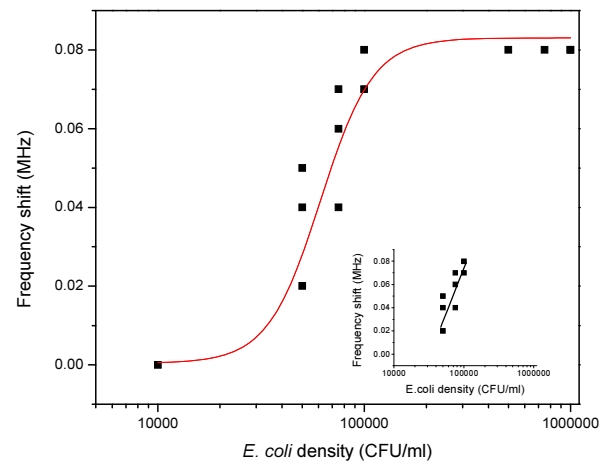

Fig. 5. Resonance frequency shift of different $E$. coli concentration with $62500 \mu \mathrm{m}^{2} \mathrm{PZT}$ sensor.

minimized, respectively. The resonant points of a ceramic resonator are basic defined as the maximum and minimum impedance at the phase of zero degree [20]. This study could not observe the resource peak by the phase result at the un-etched sensor. Nevertheless, regarding the PZT sensor after etching, the result shows the obvious resonance signal at 60 to $380 \mathrm{MHz}$ from the impedance measurement. This demonstrates that the PZT sensor via the etching process reduced a load of backside piezoelectric sensor sand increased the PZT resonance efficiency. Especially for this type, the series resonance frequency and the parallel resonance frequency could be observed at $60 \mathrm{MHz}$ and $380 \mathrm{MHz}$, respectively (as shown in Figure 4(b)). In theory calculated for gravimetric sensing, it could achieve $0.1 \mathrm{ng}$ sensing sensitivity. The detection sensitivity is higher than quartz micro-balance biosensor about one order.

\subsection{Gravimetric sensing}

In order to investigate the gravimetric sensing sensitivity of PZT sensors, it was loaded 1ul different cell numbers $E$. coli on the sensor surface. Then calculated the frequency change with the background resonance frequency was based on the LB broth (Luria-Bertani Broth) without any E. coli. The result showed the resonance frequency will be shift according to the different $E$. coli concentration which between 10000 100000 CFU/ml, as shown in Figure 5. The resonance frequency change was proportional to the E. coli concentration, and the correlation of $E$. coli concentration and resonance frequency change showed a linear relationship $\left(R^{2}=0.91\right)$. Nevertheless, the resonance frequency change of the PZT sensor was only a small change when the E. coli concentration was below 50000 $\mathrm{CFU} / \mathrm{ml}$ and above $100,000 \mathrm{CFU} / \mathrm{ml}$. In typically, the most common organism implicated in urinary tract infection (UTI) is E. coli $(80-85 \%)$ and bacteria growing at high colony counts $(100,000$ $\mathrm{CFU} / \mathrm{mL}$ ) are considered infection [21]. The results also indicated this PZT sensor could detect the $E$. coli. directly without any modification. Therefore, this sensing range of the PZT sensor has potential for application in the E. coli infection detection in the urethra in home care portable systems.

\section{Conclusion}

In this paper, the study showed that the PZT sensor via the etching process reduced the load of backside piezoelectric sensor and increased the PZT resonance efficiency. Sensing range was lower than clinical urinary tract infection, and experimental results can be successfully carried out. Current 
work is in progress to fabricate PZT biosensor that exhibit mass change range under the clinical level, so this PZT biosensor is suitable apply for preventive medicine.

\section{References}

[1] J.Z. Tsai, C.J. Chen, W.Y. Chen, J.T. Liu, C.Y. Liao and Y.M. Hsin, A new PZT piezoelectric sensor for gravimetric applications using the resonance-frequency detection, Sensor Actuator B Chem. 139 (2009), 259-264.

[2] Y. Lee, G. Lim and W. Moon, A piezoelectric micro-cantilever bio-sensor using the mass-micro-balancing technique with self-excitation, Microsyst. Technol. 13 (2007), 563-567.

[3] L. Haider, M. Gindre, D.L. Guillou-Buffelo, P. Laugier, H. Perrot, F. Carreiras and H. Darbeida, Cell attachment and spreading processes monitored by the thickness shear-mode quartz sensor, IEEE Sensors Journal 4 (2004), 535-542.

[4] H. Choi, A. Dalakoti, S. Bose and A. Bandyopadhyay, Influence of top electrode design on pMUTs performance, Sensors and Actuators A 135 (2007), 613-619.

[5] J. Xie, M. Hu, S.F. Ling and H. Du, Fabrication and characterization of piezoelectric cantilever for micro transducers, Sensors and Actuators A 126 (2006), 182-186.

[6] M. Deshpande and L. Saggere, PZT thin films for low voltage actuation: fabrication and characterization of the transverse piezoelectric coefficient, Sensor and Actuator A 135 (2007), 690-699.

[7] M.I.S. Verissimo, P.Q. Mantas, A.M.R. Senos, J.A.B.P. Oliveira and M.T.S.R. Gomes, Preparation of PZT discs for use in an acoustic wave sensor, Ceramics International 35 (2009), 617-622.

[8] H. Ishida, T. Satou, K. Tsuji, N. Kawashima, H. Takemura, Y. Kosaki, S. Shiratori and T. Agishi, The breath ammonia measurement of the hemodialysis with a QCM-NH3 sensor, Bio-Medical Materials and Engineering 18 (2008), 99-106.

[9] G. Sezonov, D. Joseleau-Petit and R. D’Ari, Escherichia coli physiology in Luria-Bertani broth, Journal of Bacteriolog. 189 (2007), 8746-8749.

[10] Z. Chen, C. Yang, B. Li, M. Sun and B. Yang, Preferred orientation controlling of PZT (52-48) thin films prepared by sol-gel process, Journal of Crystal Growth 285 (2005), 627-632.

[11] W. Gong, J.F. Li, X. Chu and L. Li, Effect of pyrolysis temperature on preferential orientation and electrical properties of sol-gel derived lead zirconate titanate films, Journal of the European Ceramic Society 24 (2004), 2977-2982.

[12] T. Kobayshi, M. Ichiki, J. Tsaur and R. Maeda, Effect of multi-coating process on the orientation and microstructure of lead zirconate titanate (PZT) thin films derived by chemical solution deposition, Thin Solid Films 489 (2005), 74-78.

[13] S. Y. Chen, Texture evolution and electrical properties of oriented PZT thin films, Materials Chemistry and Physics $\mathbf{4 5}$ (1996), 159-162.

[14] R. Thomas, S. Mochizuki, T. Mihara and T. Ishida, Effect of substrate temperature on the crystallization of $\mathrm{Pb}(\mathrm{Zr}, \mathrm{Ti}) \mathrm{O}_{3}$ films on $\mathrm{Pt} / \mathrm{Ti} / \mathrm{Si}$ substrates prepared by radio frequency magnetron sputtering with a stoichiometric oxide target, Materials Science and Engineering B 95 (2002), 36-42.

[15] S.M. Ha, D.H. Kim, H.H. Park and T.S. Kim, Crystallization and ferroelectric behavior of sputter deposited PZT using a target containing excess $\mathrm{Pb}$ and O contents, Thin Solid Films 355 (1999), 525-530.

[16] D. Damjanovic, Ferroelectric, dielectric and piezoelectric properties of ferroelectric thin films and ceramics, Rep. Prog. Phys. 61 (1998), 1267-1324.

[17] K.R. Udayakumar, P.J. Schuele, J. Chen, S.B. Krupanidhi and L.E. Cross, Thickness-dependent electrical characteristics of lead zirconate titanate thin films, J. Appl. Phys. 77 (1995), 3981-3986.

[18] B.H. Park, Y. Gim, Y. Fan and Q.X. Jia, High nonlinearity of $\mathrm{Ba}_{0.6} \mathrm{Sr}_{0.4} \mathrm{TiO}_{3}$ films heteroepitaxially grown on $\mathrm{MgO}$ substrates, Appl. Phys. Lett. 77 (2000), 2587-2589.

[19] X.D. Zhang, X.J. Meng, J.L. Sun, T. Lin, J.H. Ma and J.H. Chu, Low-temperature preparation of sputter-deposited $\mathrm{Pb}\left(\mathrm{Zr}_{0.52} \mathrm{Ti}_{0.48}\right) \mathrm{O}_{3}$ thin films through high oxygen-pressure annealing, Journal of Crystal Growth 310 (2008), $783-787$.

[20] R. Cernosek, S.J. Martin, A.R. Hillman and H.L. Bandey, Comparison of lumpedelement and transmission-line models for thicknessshear-mode quartz resonator sensors, IEEE Trans. Ultrason. Ferroelectr. Freq. Control. 45 (1998), 13991407.

[21] R. Allan, The etiology of urinary tract infection: traditional and emerging pathogens, The American Journal of Medicine 113 (2002), 14-19. 